\title{
Infectious mononucleosis: a cause of bilateral hilar lymphadenopathy
}

\author{
J.S. Friedland, G. Santis and M.J. Smith \\ Department of Thoracic Medicine, Brompton Hospital, Fulham Road, London SW3 6HP, UK.
}

\begin{abstract}
Summary: We present a case of bilateral hilar lymphadenopathy which illustrates that infection by the Epstein-Barr virus should always be considered in the differential diagnosis of this condition.
\end{abstract}

\section{Introduction}

Lymph node enlargement, both regional and generalized, is common in infectious mononucleosis. Involvement of hilar lymph nodes is described but the infrequent reports have been largely confined to the radiological literature. Furthermore, when associated with systemic symptoms it can be mistaken for sarcoidosis, lymphoma or human immunodeficiency virus (HIV) infection, particularly in younger patients. Our case report draws attention to the association of infectious mononucleosis and bilateral hilar lymphadenopathy which we believe is important and not well recognized.

\section{Case report}

A 43 year old Caucasian man presented with a 2week history of exertional dyspnoea, general malaise, sweats and myalgia. He had had no sore throat, cough, sputum or chest.pain and had been previously well. He was a smoker of 40 cigarettes per day. He had none of the risk factors for the acquired immunodeficiency syndrome.

The patient looked pale and unwell. He was pyrexial at $38^{\circ} \mathrm{C}$. Tonsillar fossa and fauces were not inflamed. Enlarged, firm, non-tender right submandibular, right axillary and bilateral inguinal lymph nodes were present. Expansion of the chest was normal. Breath sounds were vesicular with added right basal inspiratory crackles. There was no evidence of jaundice, rash, hepatosplenomegaly or neurological abnormality. Electrocardiogram was normal. Chest X-ray revealed bilateral hilar lymphadenopathy and some right basal linear shadow-

Correspondence: M.J. Smith, M.D., M.R.C.P., Upton Chest Clinic, Slough, Berks, UK.

Accepted: 27 April 1988 ing (Figure 1). At this stage the presumptive clinical diagnosis was lymphoma.

The patient's white blood cell count was markedly raised at $23.2 \times 10^{9} / 1$. Differential count showed $24 \%$ atypical monocytes, $10 \%$ monocytes, $38 \%$ lymphocytes, $27 \%$ neutrophils and $1 \%$ eosinophils. Haemoglobin, urea, electrolytes and serum angiotensin converting enzyme were all in the normal range. Deranged liver function was reflected in an alkaline phosphatase of $518 \mathrm{IU} / \mathrm{ml}$ (normal $50-200 \mathrm{IU} / \mathrm{ml}$ ), glutaryltransferase of $93 \mathrm{IU} / \mathrm{ml}$ (normal 5-28 IU $/ \mathrm{ml}$ ) and aspartate transaminase of $130 \mathrm{IU} / \mathrm{ml}$ (normal 5-17 IU/ml). Viral screens including mycoplasma and cytomegalovirus titres and toxoplasma dye test were negative. The patient was hepatitis B surface antigen-negative. Forced expiratory volume in one second was at the lower end of

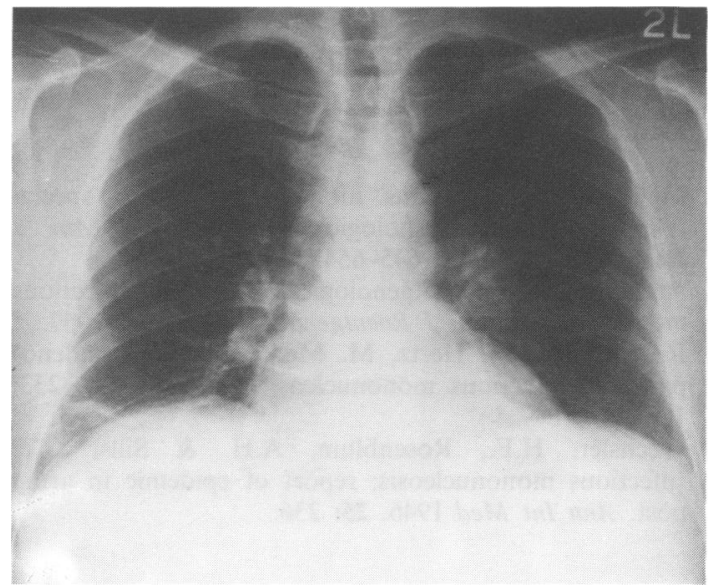

Figure 1 Chest $\mathrm{X}$-ray at presentation showing bilateral hilar lymphadenopathy and linear shadowing at right base.

(C) The Fellowship of Postgraduate Medicine, 1988 


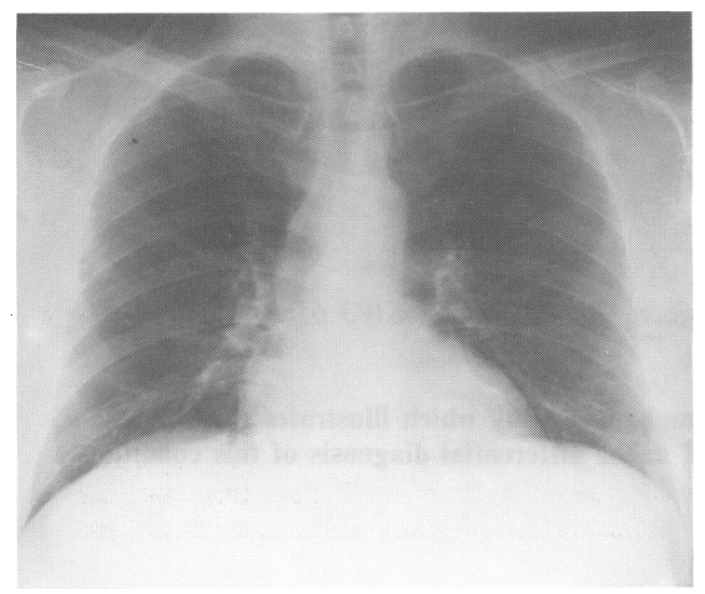

Figure 2 Repeat chest X-ray three months later showing resolution of both hilar lymphadenopathy and linear shadowing.

the normal range at $3170 \mathrm{ml}$. Forced vital capacity was reduced at $3870 \mathrm{ml}$ (predicted $4040-5470 \mathrm{ml}$ ). Transfer factor was also low at 7.49 SI (predicted 9.13-12.35 SI). The diagnosis of Epstein-Barr virus (EBV) infection was made on a positive PaulBunnell test and the detection of EBV-specific IgM by fluorescent antibody binding.

The patient improved somewhat while in hospital but full clinical recovery took 3 months. His white cell count was then $6.7 \times 10^{9} / 1$ with a normal differential count and no atypical monocytes, the PaulBunnell test was negative and EBV-specific IgM not detectable. The bilateral hilar lymphadenopathy

\section{References}

1. McCort, J.J. Infectious mononucleosis with special reference to roentgenologic manifestations. $\mathrm{Am} J$ Roentgenol 1949, 62: 645-654.

2. Arendt, J. The roentgenological aspect of infectious mononucleosis. Am J Roentgenol 1950, 64: 950-957.

3. Rosenthal, T. \& Hertz, M. Mediastinal lymphadenopathy in infectious mononucleosis. JAMA 1975, 233: 1300-1301.

4. Wechsler, H.F., Rosenblum, A.H. \& Sills, C.T. Infectious mononucleosis; report of epidemic in army post. Ann Int Med 1946, 25: 236. and right basal changes on chest X-ray have completely resolved (Figure 2).

\section{Discussion}

Epstein-Barr virus commonly causes generalized lymphadenopathy but reports of hilar lymphadenopathy associated with the virus have been infrequent and largely confined to the radiological literature. ${ }^{1,2}$ Rosenthal and $\mathrm{Hertz}^{3}$ described two cases of mediastinal lymphadenopathy among 32 patients wth infectious mononucleosis who had chest X-rays taken, and it was also present in five of 43 patients reported by McCort. ${ }^{1}$ The true frequency is not known since few patients with infectious mononucleosis receive chest $\mathrm{X}$-rays.

Interstitial pulmonary shadowing has also been described occurring alone ${ }^{4}$ or, as in our case, accompanying mediastinal lymphadenopathy. Of interest in this respect is the mild restrictive defect and reduced gas transfer in our patient. A fatal case of infectious mononucleosis described by Zeigler ${ }^{5}$ revealed interstitial and perivascular foci of mononuclear cells scattered throughout the lungs at autopsy.

The respiratory tract is clearly recognized as a reservoir for $\mathrm{EBV}^{6}$ and its association with disease of the upper airways is well established in nasopharyngeal carcinoma. Infection causing tracheal obstruction secondary to enlargement of Waldeyer's ring has also been described. ${ }^{7}$ This case report draws attention to the fact that EBV infection may also affect the lower respiratory tract and must be considered in the differential diagnosis of bilateral hilar lymphadenopathy.

5. Zeigler, E.E. Infectious mononucleosis; report of a fatal case with autopsy. Arch Pathol 1977, 37: 196-201.

6. Lung, M.L., So, S.Y., Chan, K.H., Lam, W.K., Lam, W.P. \& Ng, M.H. Evidence that respiratory tract is major reservoir for Epstein-Barr virus. Lancet 1985, i: 889-892.

7. Catling, S.J., Asbury, A.J. \& Latif, M. Airway obstruction in infectious mononucleosis. Anaesthesia 1984, 39: 699-702. 